\title{
Discovery of the first resolved triple white dwarf
}

\author{
M. Perpinyà-Vallès ${ }^{1,2}$, A. Rebassa-Mansergas ${ }^{1,3}$, B. T. Gänsicke ${ }^{2}$, S. Toonen ${ }^{4}$, \\ J. J. Hermes ${ }^{5 \star}$, N. P. Gentile Fusillo ${ }^{2}$, P.-E. Tremblay ${ }^{2}$ \\ ${ }^{1}$ Departament de Física, Universitat Politècnica de Catalunya, c/Esteve Terrades 5, 08860 Castelldefels, Spain \\ ${ }^{2}$ Department of Physics, University of Warwick, Coventry CV4 7AL, UK \\ ${ }^{3}$ Institut d'Estudis Espacials de Catalunya, Ed. Nexus-201, c/Gran Capità 2-4, 08034 Barcelona, Spain \\ ${ }^{4}$ Anton Pannekoek Institute for Astronomy, University of Amsterdam, 1090 GE Amsterdam, The Netherlands \\ ${ }^{5}$ Department of Physics and Astronomy, University of North Carolina, Chapel Hill, NC 27599, USA
}

Accepted XXX. Received YYY; in original form ZZZ

\begin{abstract}
We report the discovery of J1953-1019, the first resolved triple white dwarf system. The triplet consists of an inner white dwarf binary and a wider companion. Using Gaia DR2 photometry and astrometry combined with our follow-up spectroscopy, we derive effective temperatures, surface gravities, masses and cooling ages of the three components. All three white dwarfs have pure-hydrogen (DA) atmospheres, masses of $0.60-0.63 \mathrm{M}_{\odot}$ and cooling ages of $40-290 \mathrm{Myr}$. We adopt eight initial-to-final mass relations to estimate the main sequence progenitor masses (which we find to be similar for the three components, 1.6-2.6 $\mathrm{M}_{\odot}$ ) and lifetimes. The differences between the derived cooling times and main sequence lifetimes agree for most of the adopted initial-to-final mass relations, hence the three white dwarfs in J1953-1019 are consistent with coeval evolution. Furthermore, we calculate the projected orbital separations of the inner white dwarf binary $(303.25 \pm 0.01 \mathrm{au})$ and of the centre of mass of the inner binary and the outer companion (6 398.97 $\pm 0.09 \mathrm{au})$. From these values, and taking into account a wide range of possible configurations for the triplet to be currently dynamically stable, we analyse the future evolution of the system. We find that a collision between the two inner white dwarfs due to Lidov-Kozai oscillations is unlikely, though if it occurs it could result in a sub-Chandrasekhar Type Ia supernova explosion.
\end{abstract}

Key words: (stars:) white dwarfs; (stars:) binaries (including multiple): close; stars: individual: J1953-1019

\section{INTRODUCTION}

White dwarfs are the remnants of main sequence stars of masses up to $\simeq 8-10 \mathrm{M}_{\odot}$ (Althaus et al. 2010). Once all the nuclear burning phases that drive the evolution of these low-to-intermediate mass main sequence stars come to an end, they lose their outer layers by stellar winds or stellar interactions leaving behind their remnant hot, compact cores after the planetary nebula phase. These inert hot stellar remnants, supported by the pressure of the degenerate electrons in their interiors, then enter the white dwarf cooling sequence where their physical characteristics can be studied in detail. The gravothermal cooling process of white dwarfs is moderately well understood (Althaus \& Benvenuto 1998; Fontaine et al. 2001; Renedo et al. 2010; Camisassa et al. 2016), which turns white dwarfs into "cosmochronome-

* Hubble Fellow ters" that can be used to, e.g., derive the age of the Galactic disk (Winget et al. 1987; Garcia-Berro et al. 1988; Gianninas et al. 2015; Kilic et al. 2017), the ages of both open and globular clusters (Calamida et al. 2008; García-Berro et al. 2010; Torres et al. 2015), or to study the kinematic properties of the Galaxy through the age-velocity dispersion relation (Anguiano et al. 2017).

White dwarfs are not only found as isolated stars but also in binary and multiple stellar systems (Toonen et al. 2017). In fact, the first two white dwarfs discovered are members of a triple (40 Eri B; Herschel 1785; Bond et al. 2017b) and a binary system (Sirius B; Bessel 1844; Bond et al. 2017a), respectively. White dwarf binaries have played a key role in constraining a wide variety of open problems in astrophysics, such as the evolution of close compact binaries (Zorotovic et al. 2010; Rebassa-Mansergas et al. 2012; Camacho et al. 2014; Zorotovic et al. 2014), the age-metallicity relation of the Galactic disk (Zhao et al. 2011; Rebassa-Mansergas et al. 


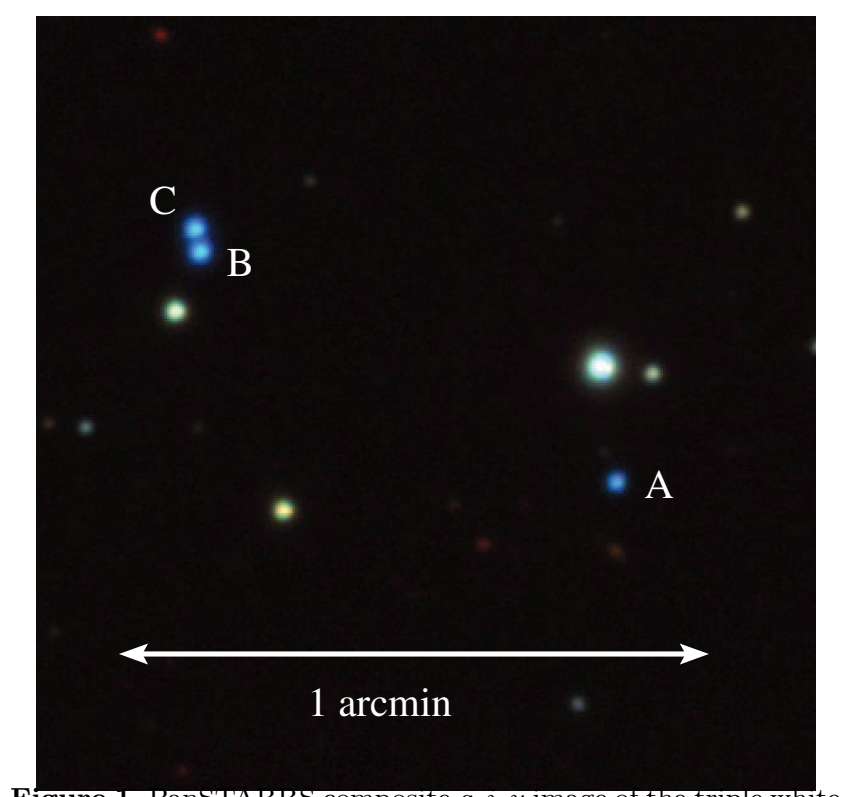

Figure 1. PanSTARRS composite $g, \imath, y$ image of the triple white dwarf system J1953-1019, components A, B, and C (see Table 1) are labelled.

2016), the age-rotation-activity relation of low-mas main sequence stars (Rebassa-Mansergas et al. 2013; Skinner et al. 2017), the pairing function of main sequence binaries (Ferrario 2012; Cojocaru et al. 2017), and the initial-tofinal mass relation (Catalán et al. 2008; Girven et al. 2010; Zhao et al. 2012; Baxter et al. 2014; Andrews et al. 2015). Last, but not least white dwarf binaries are the progenitors of type Ia supernovae (SN Ia; Wang \& Han 2012; Maoz et al. 2014; Soker 2018), which are key distance beacons for cosmological studies.

White dwarfs in multiple (at least three components) systems are relatively rare compared to those in binaries. In the solar neighbourhood (within $20 \mathrm{pc}$ ), only nine such systems have been so far identified (see Toonen et al. 2017, and references therein), and all of them contain at least one nondegenerate component. The only systems currently known that contain three compact objects are PSRJ0337+1715 (Ransom et al. 2014), which is composed of two white dwarfs and a millisecond pulsar, and WD1704+481 (Maxted et al. 2000), composed of a short-period (0.15 days) unresolved double-degenerate and a tertiary white dwarf.

Double white dwarfs that are the inner members of hierarchical triple systems provide another pathway towards SN Ia, as the tertiary can drive Lidov-Kozai oscillations that result into their collision (Benz et al. 1989; Katz \& Dong 2012). It is worth noting, however, that the expected SN Ia rate from triples is orders of magnitude below the observed rate (Toonen et al. 2018), which implies white dwarf collisions in triple systems are unlikely to be the dominant channel for producing such explosions. Lidov-Kozai driven mergers of white dwarf triples could explain a few odd white dwarf binaries in which the more massive component is hotter / looks younger (see Ferrario et al. 1997; Andrews et al. 2016).

In this paper we report the discovery of J1953-1019, the first resolved triple system formed by three white dwarfs.

\section{IDENTIFICATION OF J1953-1019}

We discovered the resolved triple white dwarf J1953-1019 as part of a search for double white dwarfs in common proper motion pairs. We performed this search by crossmatching 57201 objects with a probability of being a white dwarf greater than 0.5 from the Gentile Fusillo et al. (2015) catalogue of Sloan Digital Sky Survey (SDSS) photometric white dwarf candidates, with the Gaia Data Release 2 (DR2, Lindegren et al. 2018). The Gaia astrometry unambiguously allows us to identify the genuine white dwarfs among the initial list of candidates, and the cross-match provided us with parallaxes, proper motions, and both ugriz and Gaia photometry for a large sample of high-confidence white dwarfs. Given that white dwarfs are nearby low-luminosity objects with correspondingly large proper motions, care has to be taken in the cross-match as the Gaia and SDSS observations may have been obtained over a decade apart. Thus, we divided our cross-matching procedure in three separate steps. For each SDSS white dwarf candidate we first retrieved every matching Gaia source within a radius of $30^{\prime \prime}$ (typically four to eight objects). We then used the Gaia proper motions to "backward project" the epoch 2015.5 Gaia coordinates to the epoch of the SDSS observation. We considered a true match to be the closest Gaia source with "backward projected" coordinates within $2^{\prime \prime}$ of the SDSS ones. For each white dwarf with a true match, we then selected all Gaia sources within $2^{\prime}$, which resulted in groups of typically two and up to ten Gaia targets. Within each group we searched for white dwarfs with similar $(1 \sigma)$ parallaxes and $(5 \sigma)$ proper motions. We allowed proper motions to agree within a larger error to account for radial and/or orbital motions that would have made our white dwarf common-proper-motion pair candidates in each group seem unbound rather than co-moving objects.

This search led to the discovery of a single white dwarf triple, J1953-1019. The PanSTARRS image of J1953-1019 clearly reveals the three white dwarfs as blue stars (Fig. 1). We provide the Gaia DR2 coordinates, parallaxes, proper motions and $G, B_{\mathrm{p}}, R_{\mathrm{p}}$ magnitudes as well as the SDSS $g$ magnitudes of the three components in Table 1.

\section{SPECTROSCOPY AND STELLAR PARAMETERS}

We obtained spectroscopic follow-up of all three white dwarf components of the triple system J1953-1019 on the night of 2018 May 5 using the Goodman spectrograph (Clemens et al. 2004) mounted on the 4.1-m Southern Astrophysical Research (SOAR) telescope at Cerro Pachón in Chile. The observations were obtained under photometric conditions and $\simeq 1.2^{\prime \prime}$ seeing, using a 930 line $\mathrm{mm}^{-1}$ grating and a custom grating and camera angle setup that covers the wavelength range $\simeq 3600-5200 \AA$.

Our spectroscopy of the outer tertiary (component A, see Table 1) was obtained through a $3.2^{\prime \prime}$ slit in order to maximize signal-to-noise $(\mathrm{S} / \mathrm{N})$ ratio. We obtained $11 \times 300 \mathrm{~s}$ exposures with a resolution of roughly $3.2 \AA$ (set by the $1.2^{\prime \prime}$ seeing), achieving $\mathrm{S} / \mathrm{N}=77$ per resolution element in the continuum at $4600 \AA$.

We subsequently used a smaller $1.0^{\prime \prime}$ slit to observe the 
Table 1. Gaia coordinates, parallaxes, proper motions, $G, B_{\mathrm{p}}, R_{\mathrm{p}}$ and SDSS $g$ magnitudes of the three white dwarfs in the triple system J1953-1019. Masses, effective temperatures, surface gravities and cooling ages are also indicated, derived from SOAR spectroscopy. The error bars include external data reduction uncertainties of $1.2 \%$ in $T_{\text {eff }}$ and 0.038 dex in $\log g$ (Liebert et al. 2005). The most plausible range of white dwarf progenitor masses for each component are given in the last row.

\begin{tabular}{cccc}
\hline \hline J1953-1019 & Component A & Component B & Component C \\
\hline Gaia DR2 source ID & 4190500054845023488 & 4190499986125543168 & 4190499986125543296 \\
RA (2015.5) & 195333.11 & 195335.99 & 195336.02 \\
DEC (2015.5) & -101955.10 & -101931.76 & -101929.49 \\
$\varpi[\mathrm{mas}]$ & $7.79 \pm 0.15$ & $7.67 \pm 0.12$ & $7.64 \pm 0.12$ \\
Distance [pc] & $128.36 \pm 2.40$ & $130.37 \pm 2.11$ & $130.89 \pm 2.01$ \\
p.m. RA [mas yr $\left.{ }^{-1}\right]$ & $-10.81 \pm 0.25$ & $-11.54 \pm 0.18$ & $-10.94 \pm 0.19$ \\
p.m. DEC [mas yr $\left.{ }^{-1}\right]$ & $-16.10 \pm 0.15$ & $-16.33 \pm 0.11$ & $-15.66 \pm 0.12$ \\
$G[\mathrm{mag}]$ & 17.28 & 16.35 & 16.44 \\
$B_{\mathrm{p}}[\mathrm{mag}]$ & 17.27 & 16.05 & 16.29 \\
$R_{\mathrm{p}}[\mathrm{mag}]$ & 17.30 & 16.30 & 16.44 \\
$g[\mathrm{mag}]$ & 17.18 & 16.15 & 16.24 \\
\hline Masses $[\mathrm{M} \odot]$ & $0.63 \pm 0.03$ & $0.62 \pm 0.03$ & $0.60 \pm 0.03$ \\
$T_{\text {eff }}[\mathrm{K}]$ & $13715 \pm 310$ & $22223 \pm 360$ & $22104 \pm 350$ \\
log(g) $[\mathrm{dex}]$ & $8.03 \pm 0.05$ & $7.98 \pm 0.05$ & $7.95 \pm 0.05$ \\
Cooling age $[\mathrm{Gyr}]$ & $0.29 \pm 0.04$ & $0.042 \pm 0.008$ & $0.040 \pm 0.008$ \\
\hline
\end{tabular}

inner pair, rotating the position angle to $120 \mathrm{deg}$ so that we could obtain a spectrum of each object individually. The narrower slit also slightly improved the spectral resolution to $\simeq 2.8 \AA$. We observed the northern component $(\mathrm{C})$ with $4 \times 300$ s exposures $(\mathrm{S} / \mathrm{N}=108)$, and the southern component (B) with $3 \times 300 \mathrm{~s}$ exposures $(\mathrm{S} / \mathrm{N}=97)$. The observations of the southern component were immediately followed by an iron-argon arc lamp for wavelength calibration; the data collected with the $3.2^{\prime \prime}$ slit suffer from a large wavelength offset.

All spectra were flux calibrated using the standard star EG 274, and were bias- and flat-fielded using the software packages PAMELA and MOLLY (Marsh 1989). We also applied an absolute flux calibration to account for slit losses by normalizing the spectra to their observed SDSS $g$-band magnitudes. The spectra of the three white dwarfs revealed the typical broad absorption Balmer lines of DA white dwarfs.

We used the fitting routine outlined in Bergeron et al. (1992) to derive the surface gravities and effective temperatures of the three white dwarfs from the SOAR spectra. To that end we used the white dwarf model atmosphere spectra of Tremblay et al. (2011), including the Stark broadening tables of Tremblay \& Bergeron (2009). Given that 1D white dwarf model spectra such as those used in this work yield overestimated surface gravity values for white dwarfs of effective temperatures below $\simeq 13000 \mathrm{~K}$ (Koester et al. 2009), we compared our best-fitted effective temperature and surface gravity values to those obtained after applying the the 3D corrections of Tremblay et al. (2013) and found no difference. Fig. 2 shows the SOAR spectra along with the best-fit models and the fit parameters are reported in Table 1. We then derived the masses and cooling ages (also provided in Table 1) interpolating the surface gravities and effective temperatures in the cooling sequences of Fontaine et al. (2001) for thick hydrogen layers and C/Ocores. We compared the spectroscopic values thus obtained to those independently and photometrically derived for the three white dwarfs from the Gaia parallaxes and photometry by Jiménez-Esteban et al. (2018) and Gentile Fusillo et al. (2018) and found the values to agree within $\simeq 2.5 \sigma\left(T_{\text {eff }}\right)$, $\simeq 0.3 \sigma$ (log g) and $\simeq 0.3 \sigma$ (mass), where $\sigma$ is defined as

$\sigma=\frac{\mid \text { Spec }_{\text {value }}-\text { Phot }_{\text {value }} \mid}{\sqrt{\text { Specerror }^{2}+\text { Photerror }^{2}}}$,

and $\mathrm{Spec}_{\text {value }}$, $\mathrm{Spec}_{\text {error }}$, Phot ${ }_{\text {value}}$, Photerr are the spectroscopic and photometric stellar parameter values and their errors.

\section{PROGENITOR MASSES}

It has been shown that wide white dwarf binaries can be used to constrain the initial-to-final mass relation, since the difference between white dwarf cooling ages gives an indication of the difference between progenitor lifetimes, assuming the binary components are coeval (Finley \& Koester 1997; Girven et al. 2010; Baxter et al. 2014; Andrews et al. 2015). In this section we test if the parameters we derive for J1953-1019 are consistent with coeval evolution, when adopting the initial-to-final mass relations of Ferrario et al. (2005) (their non-linear relation), Catalán et al. (2008) (their two-piece relation), Kalirai et al. (2008), Williams et al. (2009), Salaris et al. (2009) (their linear relation), Renedo et al. (2010), Gesicki et al. (2014) (their equation 15) and Cummings et al. (2018) (their MIST-based relation).

From the above relations we first obtained the progenitor masses of the three components, where the final masses are the white dwarf masses reported in Table 1. In this exercise we took into account the white dwarf mass uncertainties, hence we obtained a possible range of progenitor masses from each relation. The results are given in Table 2. For each range of progenitor masses we then derived the corresponding main sequence lifetimes using a main sequence lifetime-mass relation. These values are also provided in Table 2 . We obtained a main sequence lifetime-mass relation by 


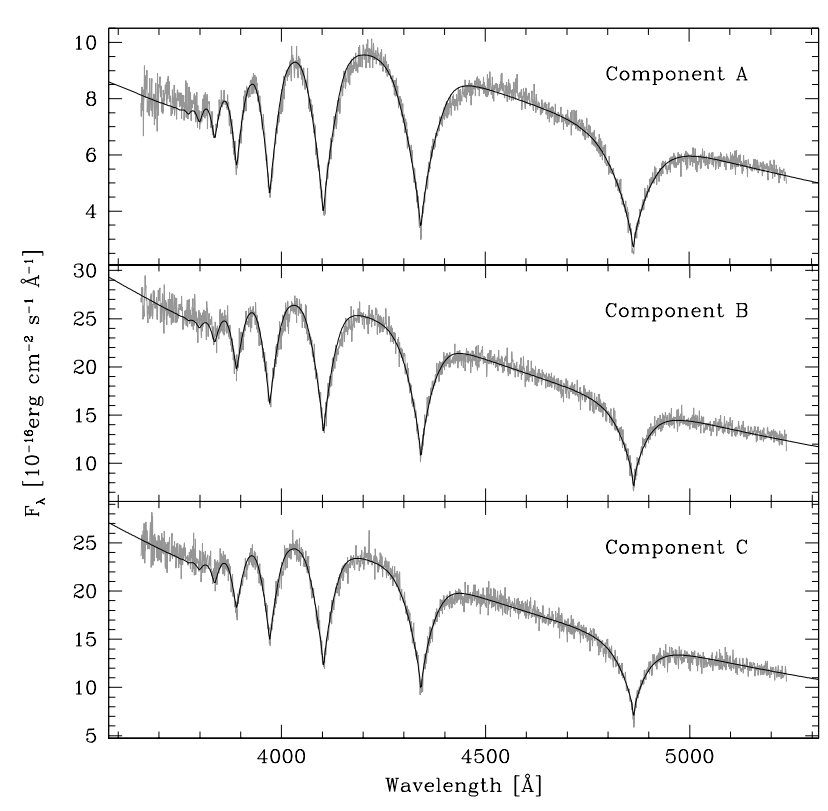

Figure 2. Best-fit models (black, see Table 1) to the SOAR spectra (gray) of the three DA white dwarfs in the triple system J1953-1019.

employing the main sequence stellar evolutionary tracks of Choi et al. (2016), where we adopted solar metallicities and rotation at 0.4 of critical velocity (see also Dotter 2016). Specifically, we computed tracks for masses ranging from $1 \mathrm{M}_{\odot}$ to $10 \mathrm{M}_{\odot}$ in steps of $0.1 \mathrm{M}_{\odot}$, which translated into main sequence lifetimes from $\simeq 0.1 \mathrm{Myr}$ to $\simeq 10 \mathrm{Gyr}$. From each track we extracted the time (i.e. age or main sequence lifetime) and the mass of the star corresponding to the transition into a white dwarf, therefore having a function relating the main sequence lifetime and the main sequence mass.

From all the possible values obtained by computing $t_{\mathrm{MS}_{\mathrm{A}}}-t_{\mathrm{MS}_{\mathrm{B}}}, t_{\mathrm{MS}_{\mathrm{A}}}-t_{\mathrm{MS}_{\mathrm{C}}}$ and $t_{\mathrm{MS}_{\mathrm{B}}}-t_{\mathrm{MS}_{\mathrm{C}}}$ (where $t_{\mathrm{MS}_{\mathrm{A}}}$, $t_{\mathrm{MS}_{\mathrm{B}}}$ and $t_{\mathrm{MS}_{\mathrm{C}}}$ are the main sequence lifetimes reported in Table 2), we kept the maximum and the minimum in each case, which defined the maximum and minimum differences between the progenitor lifetimes of the three components. These differences are compared to the difference in white dwarf cooling times in Fig. 3 for each of our adopted initialto-final mass relations.

Inspection of the top panel of Fig. 3 reveals that the difference in cooling ages and main sequence lifetimes between components $\mathrm{A}$ and $\mathrm{B}$ agree when considering all initial-tofinal mass relations. The same is true in the middle panel of the same figure when comparing the time differences between the $\mathrm{A}$ and $\mathrm{C}$ components, except for the Catalán et al. (2008), Renedo et al. (2010) and Cummings et al. (2018) relations. The bottom panel of Fig. 3 indicates that, for components $\mathrm{B}$ and $\mathrm{C}$, all the considered relations provide differences in main sequence lifetimes which are also in agreement with those obtained from the cooling times.

The above exercise shows that the masses and cooling ages of the three white dwarfs in J1953-1019 are consistent with a coeval evolution when we consider the initial-tofinal mass relations of Ferrario et al. (2005), Kalirai et al. (2008), Williams et al. (2009), Salaris et al. (2009), and
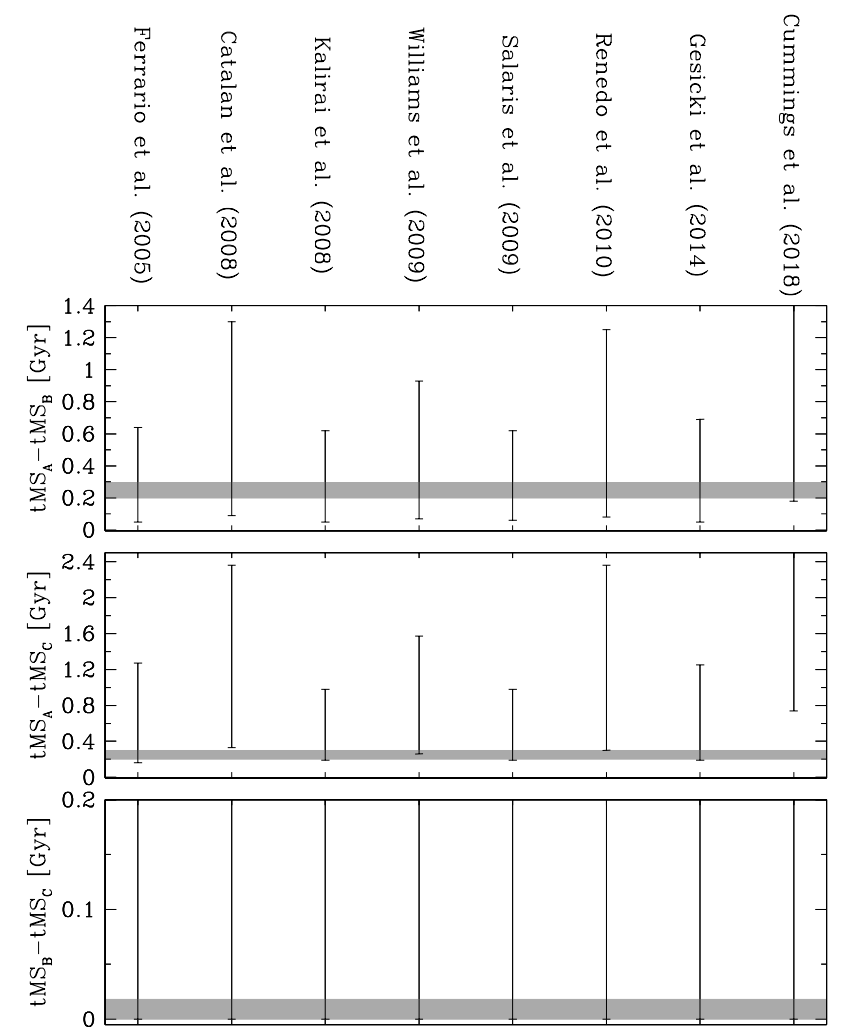

Figure 3. The possible range of values (black vertical lines) we derive for the difference between the main sequence progenitor lifetimes (obtained assuming eight different initial-to-final mass relations) as compared to the difference in cooling times implied by the atmospheric parameters that we derived from the SOAR spectroscopy, indicated by shaded grey regions. The top, middle and bottom panels illustrate the time differences between components $\mathrm{A}-\mathrm{B}\left(t_{\mathrm{MS}_{\mathrm{A}}}-t_{\mathrm{MS}_{\mathrm{B}}}\right), \mathrm{A}-\mathrm{C}\left(t_{\mathrm{MS}_{\mathrm{A}}}-t_{\mathrm{MS}_{\mathrm{C}}}\right)$ and $\mathrm{B}-$ $\mathrm{C}\left(t_{\mathrm{MS}_{\mathrm{B}}}-t_{\mathrm{MS}_{\mathrm{C}}}\right)$, respectively. Note that the difference in cooling ages is very small between components $\mathrm{B}$ and $\mathrm{C}$, we hence zoomin to this region in the bottom panel, and consequently the ranges derived from the initial-to-final mass relations extend off the scale.

Gesicki et al. (2014). The most plausible white dwarf progenitor masses should be within the ranges given in Table 2 for these relations, i.e. 1.90-2.61 $\mathrm{M}_{\odot}$ for component $\mathrm{A}, 1.80$ $2.53 \mathrm{M}_{\odot}$ for component $\mathrm{B}$ and $1.61-2.36 \mathrm{M}_{\odot}$ for component $\mathrm{C}$. These most plausible mass ranges for the individual initial-to-final mass relations are provided in Table 1.

It is important to emphasise that our aim here is to check whether or not the stellar parameters we derived for the three white dwarfs in the triple system J1953-1019 are consistent with coeval evolution and to estimate their progenitor masses. Our results should not be used to discriminate or favour a specific initial-to-final mass relation.

We also need to bear in mind that we calculated the main sequence progenitor lifetimes using the evolutionary models of Choi et al. (2016) and that the initial-to-final mass relations assumed in this work were derived adopting different evolutionary models than ours, namely those by Hurley et al. (2000); Girardi et al. (2002); Pietrinferni et al. (2004); Marigo \& Girardi (2007); Bressan et al. (2012). In order to check the impact of the choice of evolutionary model in our results we re-calculated the differences be- 
Table 2. The possible range of white dwarf progenitor masses derived adopting eight initial-to-final mass relations and taking into account the white dwarf masses (and their uncertainties) reported in Table 1. The ranges of main sequence lifetimes are also indicated.

\begin{tabular}{ccccccc}
\hline \hline IFMR & $\begin{array}{c}\text { Comp. A } \\
\text { Prog. mass } \\
{\left[\mathrm{M}_{\odot}\right]}\end{array}$ & $\begin{array}{c}\text { Comp. A } \\
\text { MS lifet. } \\
{[\mathrm{Gyr}]}\end{array}$ & $\begin{array}{c}\text { Comp. B } \\
\text { Prog. mass } \\
{\left[\mathrm{M}_{\odot}\right]}\end{array}$ & $\begin{array}{c}\text { Comp. B } \\
\text { MS lifet. } \\
{[\mathrm{Gyr}]}\end{array}$ & $\begin{array}{c}\text { Comp. C } \\
\text { Prog. mass } \\
{\left[\mathrm{M}_{\odot}\right]}\end{array}$ & $\begin{array}{c}\text { Comp. C } \\
\text { MS lifet. } \\
{[\mathrm{Gyr}]}\end{array}$ \\
\hline Ferrario et al. (2005) & $2.08-2.61$ & $0.60-1.06$ & $1.97-2.53$ & $0.65-1.24$ & $1.72-2.36$ & $0.76-1.87$ \\
Catalán et al. (2008) & $1.78-2.40$ & $0.72-1.68$ & $1.68-2.30$ & $0.81-2.02$ & $1.47-2.09$ & $1.05-3.08$ \\
Kalirai et al. (2008) & $2.02-2.49$ & $0.67-1.15$ & $1.95-2.41$ & $0.72-1.29$ & $1.79-2.26$ & $0.86-1.65$ \\
Williams et al. (2009) & $1.89-2.44$ & $0.70-1.40$ & $1.80-2.35$ & $0.77-1.63$ & $1.61-2.16$ & $0.96-2.27$ \\
Salaris et al. (2009) & $2.01-2.45$ & $0.69-1.18$ & $1.93-2.38$ & $0.75-1.31$ & $1.78-2.23$ & $0.88-1.67$ \\
Renedo et al. (2010) & $1.80-2.39$ & $0.73-1.64$ & $1.69-2.30$ & $0.81-1.98$ & $1.47-2.10$ & $1.03-3.09$ \\
Gesicki et al. (2014) & $2.01-2.53$ & $0.65-1.17$ & $1.92-2.45$ & $0.70-1.34$ & $1.71-2.27$ & $0.84-1.90$ \\
Cummings et al. (2018) & $1.39-2.14$ & $0.99-3.71$ & $1.26-2.01$ & $1.17-5.07$ & $1.01-1.76$ & $1.73-10.7$ \\
\hline
\end{tabular}

tween the progenitor lifetimes of the three white dwarfs employing these evolutionary tracks. We found that, even though the calculated progenitor lifetimes varied slightly from one model to another, the time differences were in agreement with those obtained from the cooling times for the Ferrario et al. (2005), Kalirai et al. (2008), Williams et al. (2009), Salaris et al. (2009), and Gesicki et al. (2014) initialto-final mass relations. We hence conclude the three white dwarfs in J1953-1019 are consistent with being co-eval independently of the evolutionary models used.

\section{ORBITAL PARAMETERS}

The triple system J1953-1019 consists of an inner white dwarf binary and a wider white dwarf companion (Fig. 1). We derived an angular separation of 2332.69 mas (the uncertainty is of the order of $10^{-6}$ mas) between the inner two white dwarfs from their Gaia coordinates (Table 1). To obtain the angular separation between the inner white dwarf binary and the wider companion we calculated the distance and coordinates of the centre of mass (CoM) of the inner binary $(d=130.62 \mathrm{pc} ; \mathrm{RA}=298.40003 \mathrm{deg}, \mathrm{Dec}=-10.32518$ $\mathrm{deg}$ ), which combined with the Gaia coordinates of the wider component (component $\mathrm{A}$; Table 1) resulted in an angular separation of 49222.83 mas (the uncertainty is of the order of $10^{-4} \mathrm{mas}$ ). Taking $130 \mathrm{pc}$ as the distance to the triple white dwarf (obtained simply as the weighted average of the three distances to each white dwarf component calculated from inverting the measured parallaxes), we obtained a projected separation of the inner binary of $303.25 \pm 0.01$ au and of $6398.97 \pm 0.09$ au for the inner binary and the outer companion.

Knowing the angular separations as well as the distances to each individual star and the CoM, one could attempt deriving both the true orbital separation between the inner binary and between the CoM of the inner binary and the outer companion. However, we do not proceed with this exercise for the following reason: while the Gaia positions of the two stars are extremely accurately measured by Gaia, the parallax uncertainties, though remarkably small $(\simeq 2 \%)$, are of the order of the orbital separations of the stars in J1953-1019, and do hence not constrain the orbital separations. For this reason, in what follows we only make use of the calculated projected separations.

\section{EVOLUTION}

Here we assess the formation and evolution of J1953-1019. We adopt the masses from Sect. 4 and projected separations from Sect. 5, however the system is under-constrained; that is, the eccentricities and mutual inclinations are unknown. Therefore, we take seven configurations that span the possible parameter space in which J1953-1019 is currently dynamically stable (Table 3 ). We take the projected separations as the current distance between the inner two stars, and that of the outer star to the CoM of the inner binary. For a given eccentricity of an orbit, we derive a maximum and minimum orbital separation assuming the system is currently at pericentre or apocentre. To test the dynamical stability of this three-body system, we apply the criterion of Mardling \& Aarseth (1999):

$$
\begin{aligned}
\left.\frac{a_{\text {out }}}{a_{\text {in }}}\right|_{\text {crit }}= & \frac{2.8}{1-e_{\text {out }}}\left(1-\frac{0.3 i}{\pi}\right) . \\
& \left(\frac{\left(1.0+q_{\text {out }}\right) \cdot\left(1+e_{\text {out }}\right)}{\sqrt{1-e_{\text {out }}}}\right)^{2 / 5},
\end{aligned}
$$

where $q_{\text {out }} \equiv \frac{m_{\mathrm{A}}}{m_{\mathrm{B}}+m_{\mathrm{C}}}$ with $m_{\mathrm{A}}, m_{\mathrm{B}}, m_{\mathrm{C}}$ the masses of components $\mathrm{A}, \mathrm{B}$ and $\mathrm{C}$ respectively. Furthermore $a_{\text {in }}$ and $a_{\text {out }}$ are the semi-major axis of the inner and outer orbit, and $e_{\mathrm{in}}$ and $e_{\mathrm{out}}$ the corresponding eccentricities, and $i$ is the mutual inclination. Systems are stable if $\frac{a_{\text {out }}}{a_{\text {in }}}>\left.\frac{a_{\text {out }}}{a_{\text {in }}}\right|_{\text {crit }}$. For J1953-1019 the condition for stability is met for circular orbits, but breaks down for very eccentric orbits, for example $\left[e_{\text {in }}=0, e_{\text {out }}>0.63, i=0^{\circ}\right]$ or $\left[e_{\text {in }}>0.85, e_{\text {out }}=0, i=0^{\circ}\right]$. The last configuration listed in Table 3 is dynamically stable only for retrograde orbits with mutual inclinations above $128^{\circ}$. If the system is dynamically unstable, it is likely to dissolve on a timescale of a few times the inner orbit. For this reason we only consider dynamically stable systems with $e \leq 0.5$.

Next we investigate whether the presence of the tertiary star affects the orbit of the inner binary. The lowest-order manifestation of the interaction are the Lidov-Kozai cycles (Kozai 1962; Lidov 1962), in which the eccentricity of the inner orbit and the mutual inclination varies periodically. The timescale of these cycles is (Kinoshita \& Nakai 1999):

$t_{\text {kozai }} \approx \frac{P_{\text {out }}^{2}}{P_{\text {in }}} \frac{m_{\mathrm{A}}+m_{\mathrm{B}}+m_{\mathrm{C}}}{m_{\mathrm{A}}}\left(1-e_{\text {out }}^{2}\right)^{3 / 2}$, 
where $P_{\text {in }}$ and $P_{\text {out }}$ are the periods of the inner and outer orbit, respectively. If the inner and outer orbits are circular, the Lidov-Kozai timescale is $\simeq 90 \mathrm{Myr}$. With eccentric orbits ( $e \leq 0.5$, Table 3$)$, the range of Lidov-Kozai timescales broadens ranging from a few to several hundred Myr. During the pericenter passage of the inner binary during the high-eccentricity phase of the Lidov-Kozai cycle, the inner binary can be driven towards mass transfer or a collision. This collision may result in sub-Chandrasekhar (since the total mass of the inner binary is slightly larger than $1.2 \mathrm{M}_{\odot}$ ) SN Ia explosion (e.g. Shen et al. 2017).

For the regular Lidov-Kozai cycles, the maximum amplitude in eccentricity $e_{\max }$ is strongly dependent on the unknown mutual inclination. For example, in the test-particle approximation, $e_{\max }=\sqrt{1-\frac{5}{3} \cos ^{2}\left(\mathrm{i}_{\mathrm{i}}\right)}$ (e.g. Naoz et al. 2013) and Lidov-Kozai cycles can only take place when the mutual inclination is between $39-141^{\circ}$.

The importance of higher-order terms of the three-body approximation, such as eccentric Lidov-Kozai cycles, can be quantified with the octupole parameter (Naoz 2016)

$\epsilon_{\mathrm{oct}}=\frac{m_{\mathrm{B}}-m_{\mathrm{C}}}{m_{\mathrm{B}}+m_{\mathrm{C}}} \frac{a_{\text {in }}}{a_{\mathrm{out}}} \frac{e_{\mathrm{out}}}{1-e_{\mathrm{out}}^{2}}$

As $\left|\epsilon_{\text {oct }}\right| \ll 0.01$, the eccentric Lidov-Kozai mechanism is not likely important for the majority of possible configurations of J1953-1019.

The chance for a collision to occur increases if the system is in the quasi-secular regime of dynamical evolution. In this state significant pericentre changes occur on a single orbit timescale, such that the pericentre approach can be arbitrarily close leading to physical collisions (Antonini \& Perets 2012; Katz \& Dong 2012). A triple is in the quasi-secular regime if (Antonini et al. 2014):

$\sqrt{1-e_{\text {in }}} \gtrsim 5 \pi \frac{m_{\mathrm{A}}}{m_{\mathrm{B}}+m_{\mathrm{C}}}\left(\frac{a_{\text {in }}}{a_{\text {out }}\left(1-e_{\text {out }}\right)}\right)^{3}$

For this to occur, the inner eccentricity needs to reach extremely high values during the three-body interactions; for most orbital configurations from Table $3, e_{\max } \gtrsim 0.97$, and $e_{\max } \gtrsim 0.7$ for the last system. Equivalently, if the regular Lidov-Kozai cycles are to drive the system to high eccentricities and subsequent collisions in the inner orbit, the mutual inclinations should lie between $80-110^{\circ}$ and down to $56-124^{\circ}$ for the last system.

In conclusion, there are rare, favourable conditions of the current eccentricities and mutual inclinations that make it possible for the inner binary to experience a collision due to the dynamical interaction with the tertiary white dwarf, making J1953-1019 a possible (sub-Chandrasekhar) SN Ia progenitor. Failing to ignite, this system would turn into a wide double white dwarf binary with inconsistent masses and cooling ages, akin to RE J0317-853 (Külebi et al. 2010) and HS 2220+2146 (Andrews et al. 2016). However, for the majority of the parameter space of possible orbital configurations, the inner and outer orbit of J1953-1019 are effectively decoupled.

Regarding the formation of the white dwarfs, it is likely that mass transfer has not taken place in this system and that the stars evolved into white dwarfs effectively as single stars. The reason is that if mass transfer would have taken place, the orbit would likely have been circularized due to tides, and the inner orbital separation would be equal to
Table 3. Seven possible configurations that span the possible parameter space in which J1953-1019 is currently dynamically stable and their related timescales.

\begin{tabular}{llllll}
\hline \hline$e_{\text {in }}$ & $\begin{array}{l}\text { Current } \\
\text { orbital } \\
\text { phase }\end{array}$ & $e_{\text {out }}$ & $\begin{array}{l}\text { Current } \\
\text { orbital } \\
\text { phase }\end{array}$ & $\begin{array}{l}t_{\text {kozai }} \\
(\mathrm{Myr})\end{array}$ & $\epsilon_{\text {oct }}$ \\
\hline 0 & - & 0 & - & 87 & - \\
0 & - & 0.5 & Apocentre & 26 & 0.0008 \\
0 & - & 0.5 & Pericentre & 697 & 0.0003 \\
0.5 & Apocentre & 0 & - & 103 & - \\
0.5 & Pericentre & 0 & - & 20 & - \\
0.5 & Apocentre & 0.5 & Pericentre & 831 & 0.0002 \\
0.5 & Pericentre & 0.5 & Apocentre & 6 & 0.0016 \\
\hline
\end{tabular}

the observed projected separation of $303.25 \mathrm{au}$; this is much wider than expected for post-mass transfer systems (see e.g. Toonen et al. 2014). We note that the three-body effects that potentially can lead to the collision of the white dwarfs should not be very efficient before the formation of the white dwarfs in order to avoid mass transfer. The current configuration of J1953-1019 also implies that the system has survived flybys that could have potentially led to unbinding the outer white dwarf (Antognini \& Thompson 2016).

\section{CONCLUSIONS}

We have presented the discovery of the first spatially resolved triple system composed of three white dwarfs. Followup optical spectroscopy has allowed us to derive DA spectral types of the white dwarfs, as well as to measure their effective temperatures, surface gravities, masses and cooling ages. We have adopted eight previously published initial-to-final mass relations and derived the range of possible progenitor masses for the three components and their expected main sequence lifetimes. The differences between these main sequence lifetimes and the cooling ages agree for most of the adopted initial-to-final mass relations, hence we conclude the three white dwarfs are consistent with coeval evolution. Based on the Gaia DR2 astrometry we have calculated the projected separations between the inner binary and between the centre of mass of the inner binary and the outer companion. Using these values, together with the masses of the white dwarfs, we have investigated a wide range of future evolutionary paths of the triple system. Although we cannot completely rule out the possibility for a collision between the inner binary, we consider such an event to be unlikely. A hypothetical collision could lead to a sub-Chandrasekhar Type Ia supernova explosion.

\section{ACKNOWLEDGEMENTS}

This work was supported by the MINECO Ramón y Cajal programme RYC-2016-20254, by the MINECO grant AYA2017-86274-P, by the AGAUR (SGR-661/2017), by the Netherlands Research Council NWO (grant VENI [nr. 639.041.645]), by the European Research Council under the European Union's Seventh Framework Programme 
(FP/2007-2013) / ERC Grant Agreement n. 320964 (WDTracer), by Horizon 2020 research and innovation programme n. 677706 (WD3D) and by NASA through Hubble Fellowship grant \#HST-HF2-51357.001-A, awarded by the Space Telescope Science Institute, which is operated by the Association of Universities for Research in Astronomy, Incorporated, under NASA contract NAS5-26555. Based on observations obtained at the Southern Astrophysical Research (SOAR) telescope, which is a joint project of the Ministério da Ciência, Tecnologia, e Inovação da República Federativa do Brasil, the U.S. National Optical Astronomy Observatory, the University of North Carolina at Chapel Hill, and Michigan State University.

\section{REFERENCES}

Althaus L. G., Benvenuto O. G., 1998, MNRAS, 296, 206

Althaus L. G., Córsico A. H., Isern J., García-Berro E., 2010, A\&ARv, 18, 471

Andrews J. J., Agüeros M. A., Gianninas A., Kilic M., Dhital S., Anderson S. F., 2015, ApJ, 815, 63

Andrews J. J., Agüeros M., Brown W. R., Gosnell N. M., Gianninas A., Kilic M., Koester D., 2016, ApJ, 828, 38

Anguiano B., Rebassa-Mansergas A., García-Berro E., Torres S., Freeman K. C., Zwitter T., 2017, MNRAS, 469, 2102

Antognini J. M. O., Thompson T. A., 2016, MNRAS, 456, 4219

Antonini F., Perets H. B., 2012, ApJ, 757, 27

Antonini F., Murray N., Mikkola S., 2014, ApJ, 781, 45

Baxter R. B., et al., 2014, MNRAS, 440, 3184

Benz W., Thielemann F.-K., Hills J. G., 1989, ApJ, 342, 986

Bergeron P., Saffer R. A., Liebert J., 1992, ApJ, 394, 228

Bessel F. W., 1844, MNRAS, 6, 136

Bond H. E., et al., 2017a, ApJ, 840, 70

Bond H. E., Bergeron P., Bédard A., 2017b, ApJ, 848, 16

Bressan A., Marigo P., Girardi L., Salasnich B., Dal Cero C., Rubele S., Nanni A., 2012, MNRAS, 427, 127

Calamida A., et al., 2008, ApJ, 673, L29

Camacho J., Torres S., García-Berro E., Zorotovic M., Schreiber M. R., Rebassa-Mansergas A., Nebot Gómez-Morán A., Gänsicke B. T., 2014, A\&A, 566, A86

Camisassa M. E., Althaus L. G., Córsico A. H., Vinyoles N., Serenelli A. M., Isern J., Miller Bertolami M. M., GarcíaBerro E., 2016, ApJ, 823, 158

Catalán S., Isern J., García-Berro E., Ribas I., 2008, MNRAS, 387,1693

Choi J., Dotter A., Conroy C., Cantiello M., Paxton B., Johnson B. D., 2016, ApJ, 823, 102

Clemens J. C., Crain J. A., Anderson R., 2004, in Moorwood A. F. M., Iye M., eds, Proc. SPIEVol. 5492, Ground-based Instrumentation for Astronomy. pp 331-340, doi:10.1117/12.550069

Cojocaru R., Rebassa-Mansergas A., Torres S., García-Berro E., 2017, MNRAS, 470, 1442

Cummings J. D., Kalirai J. S., Tremblay P.-E., Ramirez-Ruiz E., Choi J., 2018, ApJ, 866, 21

Dotter A., 2016, ApJS, 222, 8

Ferrario L., 2012, MNRAS, 426, 2500

Ferrario L., Vennes S., Wickramasinghe D. T., Bailey J. A., Christian D. J., 1997, 292, 205

Ferrario L., Wickramasinghe D., Liebert J., Williams K. A., 2005, doi:10.1111/j.1365-2966.2005.09244.x, 361, 1131

Finley D. S., Koester D., 1997, ApJ, 489, L79

Fontaine G., Brassard P., Bergeron P., 2001, PASP, 113, 409

Garcia-Berro E., Hernanz M., Isern J., Mochkovitch R., 1988, Nature, 333, 642

García-Berro E., et al., 2010, Nature, 465, 194
Gentile Fusillo N. P., Gänsicke B. T., Greiss S., 2015, doi:10.1093/mnras/stv120, 448, 2260

Gentile Fusillo N. P., et al., 2018, preprint, (arXiv:1807.03315)

Gesicki K., Zijlstra A. A., Hajduk M., Szyszka C., 2014, A\&A, 566, A48

Gianninas A., Curd B., Thorstensen J. R., Kilic M., Bergeron P., Andrews J. J., Canton P., Agüeros M. A., 2015, MNRAS, 449, 3966

Girardi L., Bertelli G., Bressan A., Chiosi C., Groenewegen M. A. T., Marigo P., Salasnich B., Weiss A., 2002, A\&A, 391, 195

Girven J., Gänsicke B. T., Külebi B., Steeghs D., Jordan S., Marsh T. R., Koester D., 2010, doi:10.1111/j.1365-2966.2010.16315.x, 404, 159

Herschel W., 1785, Philosophical Transactions of the Royal Society of London Series I, 75, 40

Hurley J. R., Pols O. R., Tout C. A., 2000, 315, 543

Jiménez-Esteban F. M., Torres S., Rebassa-Mansergas A., Skorobogatov G., Solano E., Cantero C., Rodrigo C., 2018, MNRAS, 480, 4505

Kalirai J. S., Hansen B. M. S., Kelson D. D., Reitzel D. B., Rich R. M., Richer H. B., 2008, ApJ, 676, 594

Katz B., Dong S., 2012, preprint, (arXiv:1211.4584)

Kilic M., Munn J. A., Harris H. C., von Hippel T., Liebert J. W., Williams K. A., Jeffery E., DeGennaro S., 2017, ApJ, 837, 162

Kinoshita H., Nakai H., 1999, Celestial Mechanics and Dynamical Astronomy, 75, 125

Koester D., Kepler S. O., Kleinman S. J., Nitta A., 2009, in Journal of Physics Conference Series. p. 012006 (arXiv: 0812.0491), doi:10.1088/1742-6596/172/1/012006

Kozai Y., 1962, AJ, 67, 591

Külebi B., Jordan S., Nelan E., Bastian U., Altmann M., 2010, A\&A, 524, A36

Lidov M. L., 1962, Planet. Space Sci., 9, 719

Liebert J., Bergeron P., Holberg J. B., 2005, 156, 47

Lindegren L., et al., 2018, A\&A, 616, A2

Maoz D., Mannucci F., Nelemans G., 2014, ARA\&A, 52, 107

Mardling R., Aarseth S., 1999, in Steves B. A., Roy A. E., eds, NATO Advanced Science Institutes (ASI) Series C Vol. 522, NATO Advanced Science Institutes (ASI) Series C. p. 385

Marigo P., Girardi L., 2007, A\&A, 469, 239

Marsh T. R., 1989, PASP, 101, 1032

Maxted P. F. L., Marsh T. R., Moran C. K. J., Han Z., 2000, 314,334

Naoz S., 2016, ARA\&A, 54, 441

Naoz S., Farr W. M., Lithwick Y., Rasio F. A., Teyssandier J., 2013, MNRAS, 431, 2155

Pietrinferni A., Cassisi S., Salaris M., Castelli F., 2004, ApJ, 612,168

Ransom S. M., et al., 2014, Nature, 505, 520

Rebassa-Mansergas A., et al., 2012, MNRAS, 423, 320

Rebassa-Mansergas A., Schreiber M. R., Gänsicke B. T., 2013, MNRAS, 429, 3570

Rebassa-Mansergas A., et al., 2016, MNRAS, 463, 1137

Renedo I., Althaus L. G., Miller Bertolami M. M., Romero A. D., Córsico A. H., Rohrmann R. D., García-Berro E., 2010, ApJ, 717,183

Salaris M., Serenelli A., Weiss A., Miller Bertolami M., 2009, ApJ, 692, 1013

Shen K. J., Toonen S., Graur O., 2017, ApJ, 851, L50

Skinner J. N., Morgan D. P., West A. A., Lépine S., Thorstensen J. R., 2017, AJ, 154, 118

Soker N., 2018, Science China Physics, Mechanics, and Astronomy, 61,49502

Toonen S., Claeys J. S. W., Mennekens N., Ruiter A. J., 2014, A\&A, 562, A14

Toonen S., Hollands M., Gänsicke B. T., Boekholt T., 2017, doi:10.1051/0004-6361/201629978, 602, A16 
Toonen S., Perets H. B., Hamers A. S., 2018, A\&A, 610, A22

Torres S., García-Berro E., Althaus L. G., Camisassa M. E., 2015, A\&A, 581, A90

Tremblay P.-E., Bergeron P., 2009, ApJ, 696, 1755

Tremblay P.-E., Bergeron P., Gianninas A., 2011, ApJ, 730, 128

Tremblay P.-E., Ludwig H.-G., Steffen M., Freytag B., 2013, A\&A, 559, A104

Wang B., Han Z., 2012, New Astron. Rev., 56, 122

Williams K. A., Bolte M., Koester D., 2009, ApJ, 693, 355

Winget D. E., Hansen C. J., Liebert J., van Horn H. M., Fontaine G., Nather R. E., Kepler S. O., Lamb D. Q., 1987, ApJ, 315, L77

Zhao J. K., Oswalt T. D., Rudkin M., Zhao G., Chen Y. Q., 2011, AJ, 141, 107

Zhao J. K., Oswalt T. D., Willson L. A., Wang Q., Zhao G., 2012, ApJ, 746, 144

Zorotovic M., Schreiber M. R., Gänsicke B. T., Nebot GómezMorán A., 2010, doi:10.1051/0004-6361/200913658, 520, A86

Zorotovic M., Schreiber M. R., García-Berro E., Camacho J., Torres S., Rebassa-Mansergas A., Gänsicke B. T., 2014, A\&A, 568, A68

This paper has been typeset from a $\mathrm{TE}_{\mathrm{E}} \mathrm{X} / \mathrm{LAT} \mathrm{EX}$ file prepared by the author. 